ఠ

\title{
Advancing personalized care in hemophilia A: ten years' experience with an advanced category antihemophilic factor prepared using a plasma/albumin-free method
}

REVIEW

\author{
Erik Berntorp' \\ Gerald Spotts ${ }^{2}$ \\ Lisa Patrone ${ }^{2}$ \\ Bruce M Ewenstein ${ }^{2}$ \\ 'Malmö Centre for Thrombosis and \\ Haemostasis, Lund University, Skåne \\ University Hospital, Malmö, Sweden; \\ ${ }^{2}$ Baxter Healthcare Corporation, \\ Westlake Village, CA, USA
}

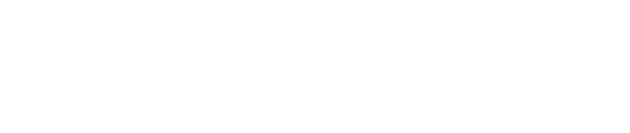

\begin{abstract}
Detailed analysis of data from studies of recombinant antihemophilic factor produced using a plasma/albumin-free method (rAHF-PFM) in previously treated patients showed a substantial level of interpatient variation in pharmacokinetics (PKs), factor VIII dosing, and annualized bleed rate (ABR), suggesting that individual patient characteristics contributed to outcome. For example, plasma half-life $\left(\mathrm{t}_{1 / 2}\right)$, recovery, and clearance appeared to differ between patients aged $<6$ years and 10-65 years. Prophylaxis resulted in lower ABRs than episodic treatment in both age groups; better adherence to the prophylactic regimen resulted in a lower ABR in patients aged 10-65 years. The weekly frequency of dosing and adherence to dosing were both significantly and inversely related to the rate of bleeding (young children, $P<0.0001$ for both all bleeds and joint bleeds; older patients, $P<0.0001$ for all bleeds and $P<0.05$ for joint bleeds), as was adherence to dosing frequency ( $P<0.0001$ for all comparisons). A postmarketing randomized study of prophylaxis demonstrated that a PK-guided dosing regimen, based on an individual patient's rAHF-PFM PK (infusion interval, estimated $t_{1 / 2}$, and recovery), was as effective as standard prophylaxis and that both prophylactic regimens were superior to episodic treatment with respect to ABR and quality of life measures. Thus, compared with standard prophylaxis, the PK-guided regimen achieved comparable efficacy with fewer weekly infusions. A two-compartment population PK model describes the PK data across the entire age range and forms the basis for future PK-guided therapy with rAHF-PFM. The model confirmed a shorter $t_{1 / 2}$ and faster clearance of rAHF-PFM in children $<6$ years of age versus patients $\geq 10$ years and predicted similar PK parameters with either a full or reduced blood sampling schedule, offering the potential for the use of PK-guided, individualized treatment in the routine clinical care setting.
\end{abstract}

Keywords: rAHF-PFM, ADVATE, FVIII, prophylaxis, pharmacokinetics, individualized

\section{Introduction}

Recombinant concentrates prepared without added animal or human plasmaderived proteins represent the current state-of-the-art product for replacement of factor VIII (FVIII) in patients with hemophilia A; they provide the highest degree of safety in terms of theoretical pathogen transmission. Although the risk of inhibitor development remains a concern, the main focus in hemophilia A therapy has now turned to the identification of optimal treatment regimens for prevention of long-term morbidity resulting from frequent bleeds, particularly in patients with moderately severe and severe disease (defined as plasma FVIII levels 1 to $2 \mathrm{IU} / \mathrm{dL}$
Correspondence: Erik Berntorp Lund University, Malmö Centre for Thrombosis and Haemostasis, Skåne University Hospital, Malmö, Sweden Tel +4640332904 Fax +4640336955

Email erik.berntorp@med.lu.se 
and $<1 \mathrm{IU} / \mathrm{dL}$ respectively). Severe hemophilia A occurs in $40 \%$ to $50 \%$ of all patients ${ }^{1,2}$ and confers high risk for both spontaneous and traumatic internal bleeding in joints, muscles, and organs. Joints that are subjected to repeated episodes of bleeding, referred to as target joints, are also at high risk for developing debilitating arthropathy. Episodic therapy, where the FVIII product is infused at the time of a bleeding episode, although effective for stopping the immediate bleed, is largely ineffective for preventing the subsequent development of arthropathy. As an alternative, Nilsson and colleagues ${ }^{3,4}$ pioneered the use of prophylactic FVIII replacement in the 1970s, with the aim of modifying severe disease to a moderate level by continual elevation of FVIII levels above $1 \mathrm{IU} / \mathrm{dL}$. Results of the Swedish studies, as well as those of numerous other studies, have proven the efficacy of prophylaxis for reducing bleeding episodes, decreasing hospitalization, and improving longterm joint function. ${ }^{5-11}$ Primary prophylaxis, started before the age of 2 years and prior to any clinically evident joint bleeding, is now the recommended treatment for children with severe hemophilia. ${ }^{8}$ Whereas the original goal of prophylactic infusion of FVIII, as noted previously, was to elevate the plasma FVIII level above $1 \mathrm{IU} / \mathrm{dL}$ in patients with severe hemophilia, the means for achieving this threshold in routine practice are not clear, and the actual required threshold for prevention of bleeding is known to vary among individuals. ${ }^{12,13}$ Thus, methodologies that would allow simple, accurate determination of appropriate prophylactic dose levels and frequencies for each individual patient could lead to significant improvement in therapy for those who currently are not reaping optimal benefit from FVIII replacement therapy.

Recombinant antihemophilic factor produced by a plasma- and albumin-free method (rAHF-PFM; ADVATE; Baxter Healthcare Corporation, Westlake Village, CA, USA) is the first advanced category recombinant FVIII (rFVIII) to be extensively studied with regard to its efficacy, pharmacokinetic (PK), and safety profile during its clinical development phase and over the 10 years since its approval in the United States. We review a series of clinical research and biostatistical modeling studies that sought to define important variables that contribute to variability of bleeding rates among patients with moderately severe to severe hemophilia A and summarize advances in tailoring therapy to patient-level rAHF-PFM pharmacodynamic and PK parameters. A schematic of key developments across the rAHF-PFM clinical development program is shown in
Figure 1; in what follows, each of the key developments will be described.

\section{Efficacy and PKs of rAHF-PFM in registration studies: identification of sources of variability

Variation in hemostatic efficacy:
importance of adherence
to dosing regimen

The hemostatic efficacy of rAHF-PFM has been demonstrated in a series of registration trials in previous treated patients with moderately severe to severe disease (Table 1), ${ }^{14-16}$ as well as in a series of post-marketing observational and interventional studies. ${ }^{17-20}$ Patients in these trials generally received a standard prophylactic regimen comprising infusion of 20 or $25-40 \mathrm{IU} / \mathrm{kg}$ at a frequency of three to four times per week, as well as episodic treatment at the time of breakthrough bleeding. Efficacy was based on the number of rAHF-PFM infusions required to ameliorate the bleeding, the overall rating of efficacy for treatment of bleeding (Table 2) and by the annualized bleed rate (ABR) during prophylaxis. In the pivotal efficacy study of patients aged $\geq 10$ years who had $\geq 150$ exposure days (ED) to FVIII, ${ }^{16}$ results demonstrated a high level of efficacy, with $93 \%$ of bleeds treated by one or two infusions of rAHF-PFM, 86\% having efficacy ratings of excellent or good, and similar results regardless of the bleed etiology (Table 1). Whereas the overall ABR during prophylaxis was 6.3 bleeds/patient/year, subjects who were less compliant with the standard prophylactic regimen had a higher bleeding rate (9.9 episodes/subject/year) than subjects who were more compliant (4.4 episodes/subject/year; Wilcoxon $P<0.03$ ), where compliance was defined as receipt of $\geq 25$ to $40 \mathrm{IU} / \mathrm{kg}$ per infusion for at least $80 \%$ of infusions and $\geq 3$ to 4 infusions per week for at least $80 \%$ of the weeks on study. Further, although $90 \%$ of prophylactic infusions were in the protocol-specified range of 20 to $50 \mathrm{IU} / \mathrm{kg}$ (doses $>40 \mathrm{IU} / \mathrm{kg}$ were permitted for periods of anticipated increased physical activity), the overall range was quite wide (9.4-110.7 $\mathrm{IU} / \mathrm{kg}$; median, $30.7 \mathrm{IU} / \mathrm{kg}$ ). The wide range suggests that some patients required higher dosing for prevention of bleeding. Dosing decisions, however, are subject to potential bias, because the actual regimen was chosen by the investigators based on their own standard of practice and/or knowledge of the patient's previous response to treatment.

Hemostatic efficacy results were similar in the longerterm rAHF-PFM continuation study, in which patients were 


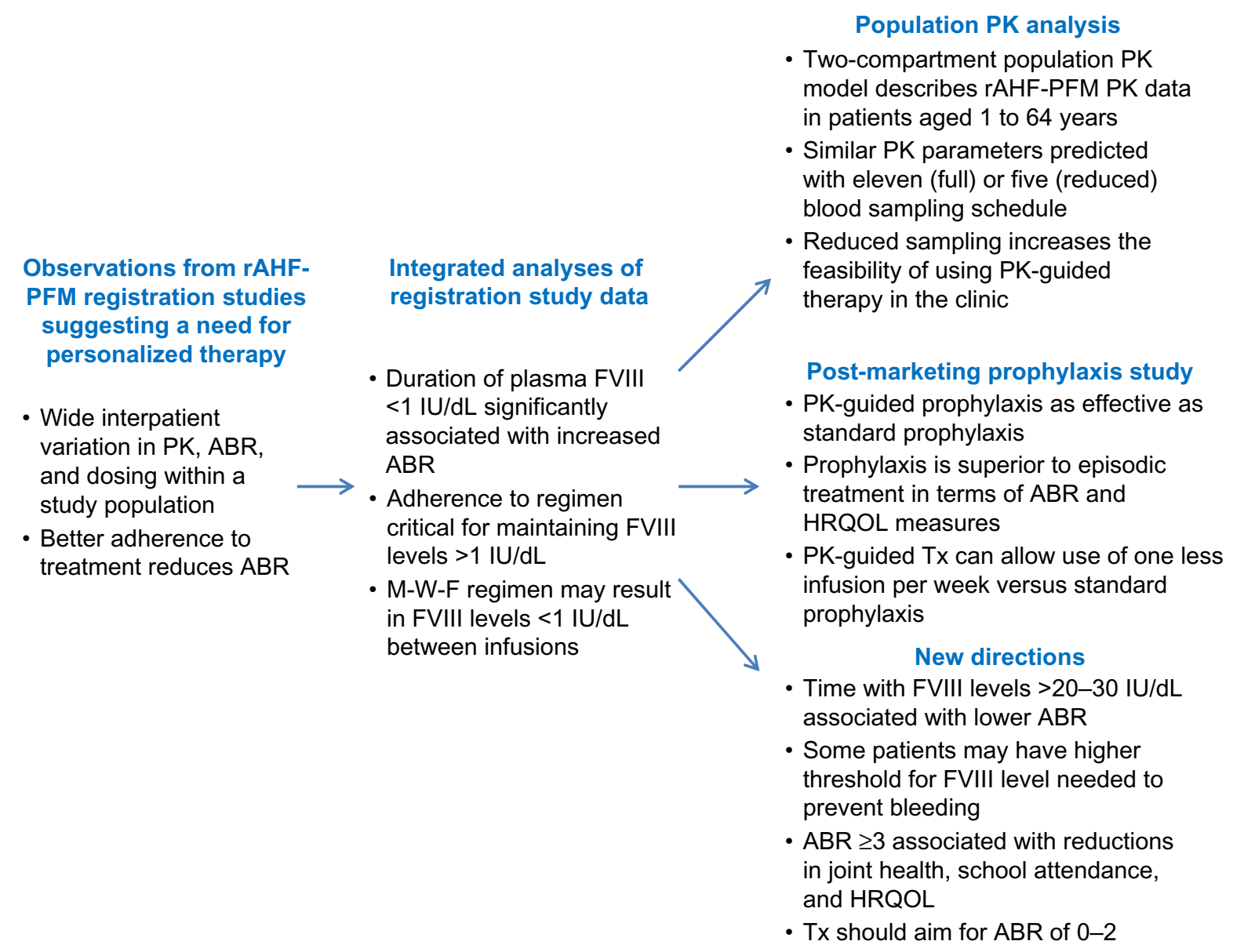

Figure I Chronology of observations from studies of rAHF-PFM leading toward personalized care for patients with hemophilia A.

Abbreviations: ABR, annual bleed rate (bleeds/patient/year); HRQOL, health-related quality of life; M-W-F, Monday-Wednesday-Friday; PK, pharmacokinetic; Tx, therapy; FVIII, factor VIII; rAHF-PFM, recombinant antihemophilic factor produced using a plasma/albumin-free method.

assigned by the investigator to one of three rAHF-PFM therapeutic regimens: standard prophylaxis as used in the pivotal study; ${ }^{16}$ modified prophylaxis, where dose and frequency were determined by the investigator; and episodic treatment at the time of a bleeding event. ${ }^{21}$ Additionally, the results showed that ABRs were markedly lower among patients treated either by standard prophylaxis ( $\mathrm{n}=54,6.0$ bleeds/subject/year) or modified prophylaxis ( $\mathrm{n}=53,4.8$ bleeds/subject/year) than for patients treated episodically ( $\mathrm{n}=9,18.5$ bleeds/patient/year). Eleven subjects, all of whom were on prophylaxis, reported no bleeding episodes during the study. Consistent with data from the pivotal study, the ABR was lower among patients who complied with the standard prophylactic regimen than that among those who were noncompliant (30 patients; 4.5 bleeds/year versus [vs] 24 patients; 7.9 bleeds/year).

An additional study demonstrated the efficacy of rAHFPFM in pediatric previously treated patients (PTPs; $<6$ years of age; $\geq 50$ EDs $).{ }^{14}$ Patients were treated on one of the three regimens described above for the continuation study, except that dosing for standard prophylaxis ranged from 25 to $50 \mathrm{IU} / \mathrm{kg}$ and prophylaxis was continued for a period of $\geq 50$ EDs or 6 months. Results showed that compared with standard prophylaxis, modified prophylaxis was characterized by fewer infusions per week at a higher median dose perinfusion (Table 1). Hemostatic efficacy results were similar to those observed in the older children, adolescents, and adults in the pivotal and continuation studies, with a markedly higher ABR for patients treated episodically than for those treated on either prophylactic regimen. Notably, although no joint bleeds occurred in patients treated on either prophylactic regimen, a median joint ABR of 14.2 bleeds/patient/year was observed for patients treated episodically.

\section{Variation in bleeding patterns in patients with severe hemophilia $A$}

The large body of data from the clinical registration trials allowed us to examine relationships between a number of variables related to treatment and bleeding and to compare 


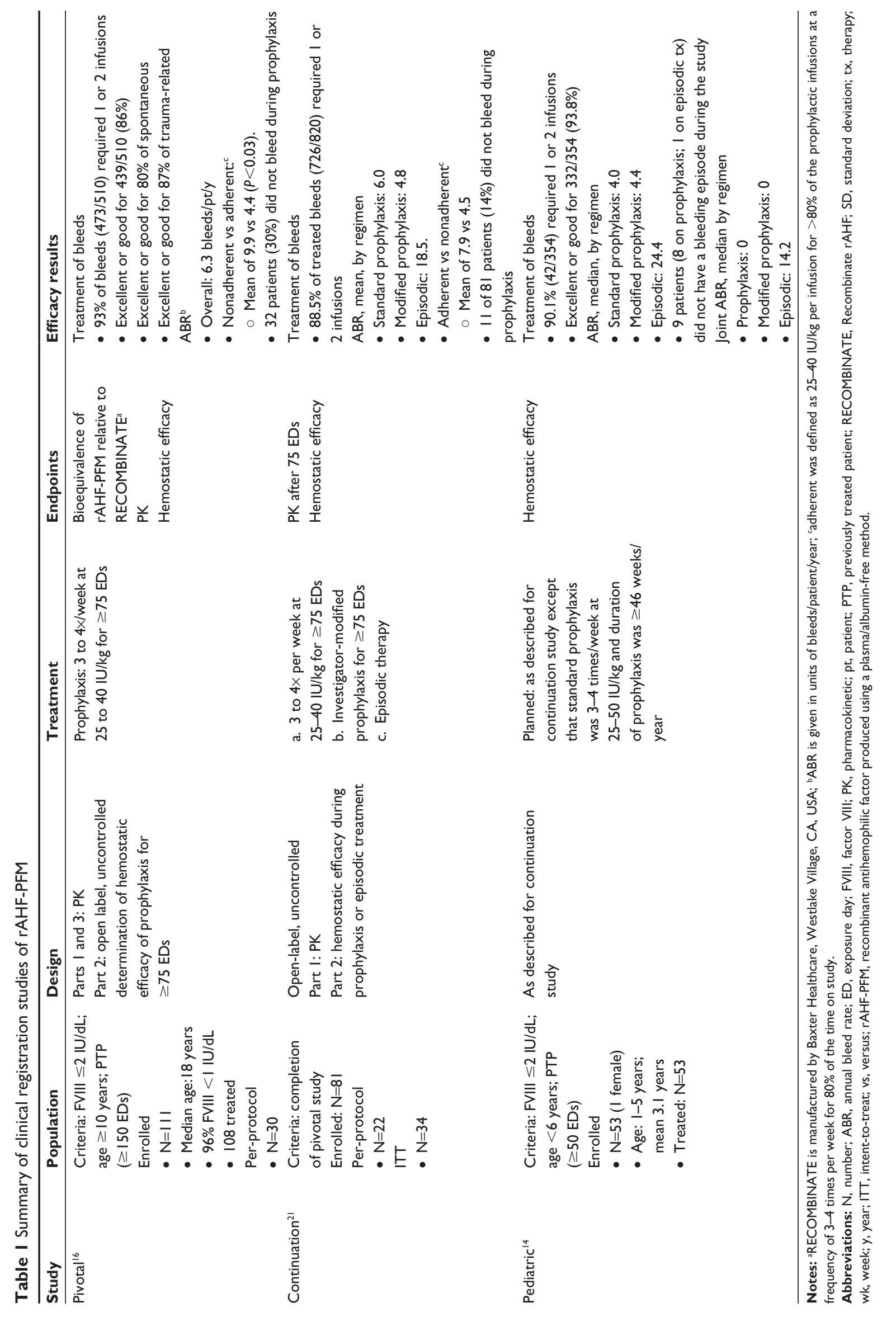


Table 2 Efficacy ratings for treatment of bleeding in studies of rAHF-PFM

\begin{tabular}{ll}
\hline Rating & Definition \\
\hline Excellent & $\begin{array}{l}\text { Abrupt pain relief and/or unequivocal } \\
\text { improvement in objective signs of bleeding } \\
\text { within approximately } 8 \text { hours after a single } \\
\text { infusion } \\
\text { Definite pain relief and/or improvement in signs } \\
\text { of bleeding within approximately } 8 \text { hours after } \\
\text { an infusion, but possibly requiring more than } \\
\text { one infusion for complete resolution } \\
\text { Probable or slight beneficial effect within } \\
\text { Fair }\end{array} \quad \begin{array}{l}\text { approximately } 8 \text { hours after the first infusion, } \\
\text { and usually requiring more than one infusion } \\
\text { No improvement, or worsening of symptoms }\end{array}$ \\
\hline
\end{tabular}

Abbreviation: rAHF-PFM, recombinant antihemophilic factor produced using a plasma/albumin-free method.

these relationships among children, adolescents, and adults. We first analyzed bleeding patterns during prophylactic treatment among the subset of all registration trial patients who had severe hemophilia $(\mathrm{n}=145) \cdot{ }^{15}$ Results of this analysis showed that mean weight-adjusted prophylactic doses decreased with increasing patient age, whereas dosing frequency was constant: $108 \mathrm{IU} / \mathrm{kg} /$ week given as $2.9 \mathrm{infu}-$ sions for children 1-6 years of age, $86 \mathrm{IU} / \mathrm{kg} /$ week given as 2.7 infusions for adolescents (10-17 years of age), and $75 \mathrm{IU} / \mathrm{kg} /$ week given as 2.6 infusions for adults $18-65$ years of age. All three age groups had low rates of breakthrough bleeding during prophylaxis: median ABRs were 3.1 bleeds/ patient/year for the young children, 3.3 bleeds/patient/year for the adolescents, and 2.1 bleeds/patient/year for the adults. The percentage of bleeds occurring in joints increased with increasing age, with joint bleeds representing $21 \%$ of all bleeds in young children, $50 \%$ of all bleeds in adolescents, and $62 \%$ of all bleeds in adults. Furthermore, the proportion of joint bleeds attributed to trauma was numerically, but not statistically higher in adolescents (51\%) than that in young children (29\%) or adults (42\%). In a multivariate analysis, arthropathy at baseline showed borderline significance $(P=0.072)$ as a risk factor for increased frequency of joint bleeds, consistent with previous analyses showing that prevention of development of target joints and arthropathy, for example with primary prophylaxis, reduced future bleeding. ${ }^{6,10,22}$ Time of day and time of year were also found to have an association with bleeding. The percentage of joint bleeds occurring in summer was higher in older patients $(43 \%$ or $46 \%)$ than it was in younger patients $(21 \%)$, and a multivariate analysis showed that after controlling for multiple factors, a significant association was observed between season and ABR for both all bleeds and joint bleeds $(P<0.01$ for both variables) among patients $10-65$ years of age. The time of day of the prophylactic infusion also significantly affected bleeding in children $<6$ years old, where the ABR for joint bleeds was significantly lower for those who received their prophylactic infusions during the morning than for those treated in the afternoon (median 0 [interquartile range (IQR) $0.0-0.4$ ] bleeds/patient/year vs 1.8 [IQR $0.0-5.2$ ] bleeds/ patient/year, respectively; $P<0.05$ ).

\section{Variation in rAHF-PFM PKs}

In the three registration studies described previously, after single $50 \mathrm{IU} / \mathrm{kg}$ doses of rAHF-PFM, $\mathrm{t}_{1 / 2}$ (plasma half-life) values varied from 6 to 25 hours in patients $\geq 10$ years of age $^{16}$ and from 6.8 to 15.4 hours in younger children. ${ }^{21}$ The wide range in $t_{1 / 2}$ is consistent with observations with other FVIII concentrates, ${ }^{23-25}$ and increasing $t_{1 / 2}$ has been correlated with increasing age in patients $\geq 10$ years in a previous study ${ }^{26}$ In the rAHF-PFM studies, the fifth, 50th, and 95th percentiles for $\mathrm{t}_{1 / 2}$ of rAHF-PFM were 7.4, 9.4, and 13.1 hours, respectively, in young children, and 7.5, 10.4 , and 16.5 hours, respectively, in patients $\geq 10$ years of age,$^{34}$ suggesting that $t_{1 / 2}$ might be somewhat shorter in children $<6$ years of age than that in older patients. Weightadjusted in vivo recovery (IVR) also appeared to be lower in the young children than in the older patients, and the $\mathrm{CL}$ appeared to be higher in the youngest children compared with the respective values for patients aged 10-65 in the pivotal study. We also found that IVR increased linearly and the volume of distribution at steady state $\left(\mathrm{V}_{\mathrm{ss}}\right)$ decreased linearly as a function of body mass index within the population of children aged $<6$ years. Mean residence time and $t_{1 / 2}$, both of which are measures of FVIII plasma persistence, had significant positive relationships with age: $t_{1 / 2}$ increased by 24 minutes/year over the age range 1-6 years. These differences in rAHF-PFM plasma PKs presumably result from physiologic differences, such as larger plasma volume for weight, larger volume of distribution due to larger extracellular, and total body water spaces, higher ratio of liver to body weight. Also, differences in von Willebrand factor levels were found in young children compared with older adults, which have been correlated with FVIII $\mathrm{t}_{1 / 2} \cdot{ }^{23}$

We also considered the possibility that some of the observed differences may have resulted from a lack of early blood sampling in the pediatric population, where the first post infusion sample was collected at 1 hour after dosing, as opposed to that in the older patient population, where three post infusion samples were collected within 
the first 1 hour after dosing (peak FVIII levels were observed within the first 30 minutes after dosing). Analysis of PK data from 52 young children and 100 patients $\geq 10$ years of age indicated that approximately half of the differences in IVR, CL, and $t_{1 / 2}$ for rAHF-PFM could be attributed to the reduced blood sampling schedule used for the young children. ${ }^{27}$ The effects of age and sampling schedule were addressed further in a subsequent study that modeled the population kinetics of rAHF-PFM, as we will now discuss. ${ }^{36}$

\section{Advances in understanding the relationships between PKs, therapy, and outcome}

A number of reports have suggested and have begun to investigate the use of patient characteristics, including FVIII PK parameters, to determine appropriate dosing of individual patients. ${ }^{12,13,26,28-30}$ Consistent with these reports, the interpatient variation in hemostatic efficacy, rates of bleeding, and rAHF-PFM PKs we observed suggest that for optimal benefit, FVIII replacement therapy should be individualized, using knowledge of a patient's PK parameters and bleed-event history, and also perhaps according to other variables such as the patient's activity levels, that might predict treatment outcome. To further understand the relationships between $\mathrm{PK}$ and bleeding frequency at the individual patient level, we next examined relationships between individual PK, FVIII trough levels, and bleeding frequency, using data combined from all three clinical registration studies of rAHF-PFM. ${ }^{14,16,21}$

\section{Linking PKs and FVIII trough levels to bleeding rates during prophylaxis}

\section{Breakthrough bleeding and FVIII trough levels}

The breakthrough bleeding that occurs in a patient being treated prophylactically is thought to result from a lower-than-expected blood FVIII level occurring between infusions, as suggested by early studies comparing weekly dosing frequencies. ${ }^{31,32}$ However, in a previous retrospective study of prophylaxis, where dosing was typically based on bleeding frequency rather than on maintenance of FVIII levels $>1 \mathrm{IU} / \mathrm{dL}$, some patients did not bleed even when the FVIII trough level was $<1 \mathrm{IU} / \mathrm{dL}$, and others bled with an FVIII trough $>3$ IU/dL. ${ }^{12}$ Additionally, a prospective study of secondary prophylaxis, ie, prophylaxis started after 2 years of age or after two or more joint bleeds in adults with severe disease, showed a wide range of FVIII trough levels. ${ }^{11}$ These data indicate that identification of appropriate dosing in individuals will require examination of multiple patient and $\mathrm{PK}$ variables.

With this in mind, Collins et $\mathrm{al}^{33}$ analyzed relationships between FVIII levels, adherence to dosing frequency, and ABR from the combined registration study patients who had received any duration of prophylaxis ( 99 patients $\geq 10$ years of age, 44 patients $<6$ years of age; minimal to maximal time on prophylaxis 194-1024 days). In that analysis, the FVIII concentration after each infusion was projected based on the infusion dose and the observed PK parameters. FVIII $\mathrm{t}_{1 / 2}$ showed a significant negative association with bleed rate $(P<0.05)$ in the young children, but not in patients $\geq 10$ years of age. Key factors predictive of bleed rate in the overall sample population were dosing frequency, adherence to dosing frequency, and the related variable of time spent with plasma FVIII $<1$ IU/dL. The observed time spent with FVIII $<1$ IU/dL (calculated on the basis of the FVIII infusions given) was compared with the predicted time each patient would have spent below these levels had they adhered to a standard Monday-Wednesday-Friday (M-W-F) prophylactic regimen of $30 \mathrm{IU} / \mathrm{kg}$. Results of this analysis showed that among older patients, the median time below $1 \mathrm{IU} / \mathrm{dL}$ would decrease from 16.5 hours to 10.0 hours if they were fully adherent. The effect of adherence on time spent with FVIII < 1 IU/dL was not observed in the younger patients.

For both age groups, ABRs for all bleeds and for joint bleeds increased as a function of duration of time with plasma FVIII $<1$ IU/dL (Figure 2). For each additional hour with FVIII $<1 \mathrm{IU} / \mathrm{dL}$, the ABR increased by $1.4 \%$ (confidence interval [CI] $0.21 \%$ to $2.62 \%$ ) in the older patients and by $2.2 \%$ (CI $1.58 \%-2.78 \%$ ) in the young children. A multivariate analysis adjusted for etiology, site, age, and weight in comparison with ideal weight showed that in both young children and in the older population, time spent with plasma FVIII $<1 \mathrm{IU} / \mathrm{dL}$ was a highly significant predictor of the rate of all bleeding ( $P<0.0001$ for young children; $P<0.05$ for older patients) and the rate of joint bleeds $(P<0.0001$ for young children; $P<0.02$ for older patients). The weekly frequency of dosing was significantly and inversely related to the rate of bleeding (young children: $P<0.0001$ for both all bleeds and joint bleeds; older patients: $P<0.005$ for all bleeds and $P<0.05$ for joint bleeds), as was adherence to dosing frequency ( $P<0.0001$ for all comparisons). Adherence to dose was not a factor because nearly all patients, when treated, received the prescribed dose. Thus, regardless of dose, lack of adherence to the prescribed frequency of infusions appeared to be the most significant variable predicting a higher bleed 
rate. The model suggested that full adherence would have resulted in approximately one fewer bleed per year in both age groups.

\section{Using PKs to predict time with FVIII level $<\mathrm{I}$ IU/dL}

The population-based findings described previously suggest that continuously maintaining plasma FVIII levels $\geq 1 \mathrm{IU} / \mathrm{dL}$ would be sufficient for prevention of breakthrough bleeding in many patients on prophylaxis. Individual patients, however, may have widely varying relationships between FVIII trough levels and risk for bleeding: whereas some patients may require FVIII levels $>1 \mathrm{IU} / \mathrm{dL}$ to prevent bleeds, others remain bleed-free with trough levels $\leq 1 \mathrm{IU} / \mathrm{dL}$. ${ }^{12,13}$ Thus, the FVIII trough level of the individual patient is dependent not only on dose and frequency of dosing, but also on the patient's PK profile, which may vary widely across the patient population. ${ }^{28}$ As shown by Collins et al, ${ }^{11}$ even when patients are receiving the same dose and frequency of FVIII replacement, the trough FVIII and duration of time with FVIII below $1 \mathrm{IU} / \mathrm{dL}$ (or other threshold) may vary markedly.

Using $1 \mathrm{IU} / \mathrm{dL}$ as a target trough level, simulations were performed to determine the effect of the observed FVIII $t_{1 / 2}$ and IVR on FVIII levels following a single $30 \mathrm{IU} / \mathrm{kg}$ infusion. ${ }^{34}$ A plot of the $\log$ (FVIII level) vs time for a patient at the fifth or 95th percentiles for $t_{1 / 2}$ (assuming median IVR) demonstrated that the duration of time before the FVIII level fell to $<1 \mathrm{IU} / \mathrm{dL}$ varied according to $t_{1 / 2}$ : those with longer $t_{1 / 2}$ took longer to reach the $1 \mathrm{IU} / \mathrm{dL}$ threshold. Among the young children, the time for FVIII to fall to $<1 \mathrm{IU} / \mathrm{dL}$ varied from 44.0 hours (fifth percentile for $t_{1 / 2}$ ) to 78.1 hours (95th percentile for $t_{1 / 2}$ ). Similarly, in the older patients, the time for FVIII to fall to $<1 \mathrm{IU} / \mathrm{dL}$ varied from 46.4 hours (fifth percentile for $t_{1 / 2}$ ) to 103.5 (95th percentile for $\mathrm{t}_{1 / 2}$ ). IVR (assuming a median $\mathrm{t}_{1 / 2}$ ) was not as strong a predictor for time to the plasma FVIII threshold, as the times varied by approximately 10 to 11 hours for the fifth and 95th IVR percentiles.

Additional simulations demonstrated that varying the $t_{1 / 2}$ from the fifth to 95th percentile (assuming average IVR) for patients treated prophylactically on an alternate-day regimen at $30 \mathrm{IU} / \mathrm{kg}$ per dose, as well as varying the dosing frequency for a constant weekly dose of $105 \mathrm{IU} / \mathrm{kg}$ (assuming average $\mathrm{t}_{1 / 2}$ and IVR), had marked effects on FVIII trough levels and the time required to reach the IU/dL threshold. For example, in both young children and older patients, the trough level was predicted to be less than the threshold for a patient with a $_{1 / 2}$ at the fifth percentile, whereas a patient at the 95 th percentile would have a trough level well above the threshold.
Similarly, daily dosing was predicted to result in trough levels well above the threshold, whereas dosing every third day resulted in trough levels below the threshold. The time to reach the $1 \mathrm{IU} / \mathrm{dL}$ threshold was approximately 45 hours for all patients with a $t_{1 / 2}$ at the fifth percentile, and varied from 61 (young children) to 70 hours (patients aged $\geq 10$ years) in patients simulated to receive infusions every third day. Patients simulated to have $t_{1 / 2}$ at or above the median, or to receive infusions daily or every other day would be expected to maintain FVIII above the $1 \mathrm{IU} / \mathrm{dL}$ threshold at all times. Simulations of alternate day infusions at doses of 10, 20, 30, or $50 \mathrm{IU} / \mathrm{kg}$ (assuming average recovery and $t_{1 / 2}$ ) showed smaller variations in FVIII trough level and predicted that only patients dosed at $10 \mathrm{IU} / \mathrm{kg}$ would experience FVIII trough levels $<1 \mathrm{IU} / \mathrm{dL}$.

A simulation of the combined effects of FVIII $t_{1 / 2}$ and dosing frequency (assuming weekly FVIII dose of $105 \mathrm{IU} / \mathrm{kg}$ and an average IVR) revealed that a patient of any age treated with daily infusions at $15 \mathrm{IU} / \mathrm{kg}$ would likely never have plasma FVIII levels $<1 \mathrm{IU} / \mathrm{dL}$. Nor would a patient treated on alternate days or every third day who had a FVIII $t_{1 / 2}$ above the median experience plasma FVIII levels below the threshold. However, the simulation also showed increasing time a patient would have plasma FVIII $<1 \mathrm{IU} / \mathrm{dL}$ with decreasing FVIII $\mathrm{t}_{1 / 2}$. Conversely, as the frequency of infusions increased, the time a patient would require to reach a plasma FVIII $<1 \mathrm{IU} / \mathrm{dL}$ level decreased. Thus, the time with FVIII below the threshold could be minimized or eliminated in many patients (ie, even those with FVIII $t_{1 / 2}$ below the median) if they received FVIII infusions more frequently, for a total weekly dose of $105 \mathrm{IU} / \mathrm{kg}$. However, the potential negative impact of daily dosing on health-related quality of life (HRQOL), ${ }^{35}$ as well as limited venous access, particularly in children, may pose significant barriers for broad patient acceptance.

We also examined the predicted combined effects of the commonly used M-W-F regimen (at doses of 20, 30, or $50 \mathrm{IU} / \mathrm{kg}$ ) and FVIII $t_{1 / 2}$ on FVIII trough levels in young children. ${ }^{34}$ Our simulation revealed that those with FVIII $t_{1 / 2}$ below 10.5 hours would experience some time with trough levels $<1 \mathrm{IU} / \mathrm{dL}$ in all three dosing scenarios. Consistent with this idea, in other patients treated on a three times per week FVIII regimen, $82.5 \%$ of breakthrough bleeds occurred between 48 and 72 hours after the last prophylactic infusion. ${ }^{11}$ The duration of time spent with FVIII below this threshold increases both with decreasing $t_{1 / 2}$ and decreasing FVIII dose, such that even at the $50 \mathrm{IU} / \mathrm{kg}$ dose level, a patient with a 9-hour $\mathrm{t}_{1 / 2}$ would experience approximately 10 hours per week with FVIII $<1$ IU/dL. As was previously reported, the ABR 
is predicted to increase by $2.2 \%$ for each hour for each week that FVIII is $<1 \mathrm{IU} / \mathrm{dL}$ in young children. ${ }^{33}$ This simulation predicted that treating a typical child $\leq 6$ years of age with the commonly used regimen of $30 \mathrm{IU} / \mathrm{kg}$ three times per week would result in $42 \%$ more bleeding episodes than if treated with the same dose on alternate days. This finding suggests that the preferred approach to maintaining FVIII above 1 IU/ $\mathrm{dL}$ (or other threshold) is to infuse more regularly, using an every-day or every-other-day schedule, rather than an M-W-F schedule. The findings from this study ${ }^{34}$ indicate that young children and older patients require different regimens for optimal prophylaxis, and that the commonly used M-W-F dosing frequency is suboptimal in both age groups unless pharmacokinetically-guided to optimize dose in patients with adequate $\mathrm{FVIII} \mathrm{t}_{1 / 2}$ characteristics.

\section{PK-guided prophylaxis}

\section{Post-marketing study of standard prophylaxis and PK-guided prophylaxis versus episodic treatment with rAHF-PFM}

To test the hypothesis that PK-guided prophylaxis is effective for preventing bleeding in children, adolescents, and adults, a prospective, randomized comparison of 12 months of PK-guided every third day prophylaxis vs standard everyother-day prophylaxis with rAHF-PFM in previously treated patients with moderately severe to severe disease, was conducted recently. ${ }^{17}$ Efficacy of both prophylactic regimens was compared with a lead-in 6-month period of episodic treatment with rAHF-PFM (Figure 3). Doses on the standard regimen were 20-40 IU/kg every other day, with the actual dose determined by the investigator. The PK-guided doses were 20-80 IU/kg every third day, with the actual dose derived using the 72-hour infusion interval, the estimated terminal $t_{1 / 2}$, and the incremental recovery ${ }^{17}$ determined during episodic treatment. The general aim of both regimens, whether based on individual PK assessment only or by weight-based dosing, was to maintain an FVIII trough level $\geq 1 \mathrm{IU} / \mathrm{dL}$.

The median (range) doses per infusions were 31.4 (11.8-80.9) IU/kg for standard prophylaxis and 43.0 (13.0-107.1) IU/kg for PK-guided prophylaxis. Whereas all patients experienced one or more bleeding episode during the 6-month episodic treatment period, 22 patients (13/32 on standard prophylaxis and 9/34 on the PK-guided regimen) did not experience any bleeding during the 12-month period of prophylaxis. In the intent-to-treat (ITT) analysis, there was no significant difference in the ABR for patients on standard prophylaxis or $\mathrm{PK}$-guided prophylaxis $(P=0.2588)$. The median ABR was significantly lower for the combined prophylaxis regimens than for episodic treatment (1.1 vs 43.9 bleeds/patient/year; $P<0.0001)$. Results were similar for joint bleeds, nonjoint bleeds,

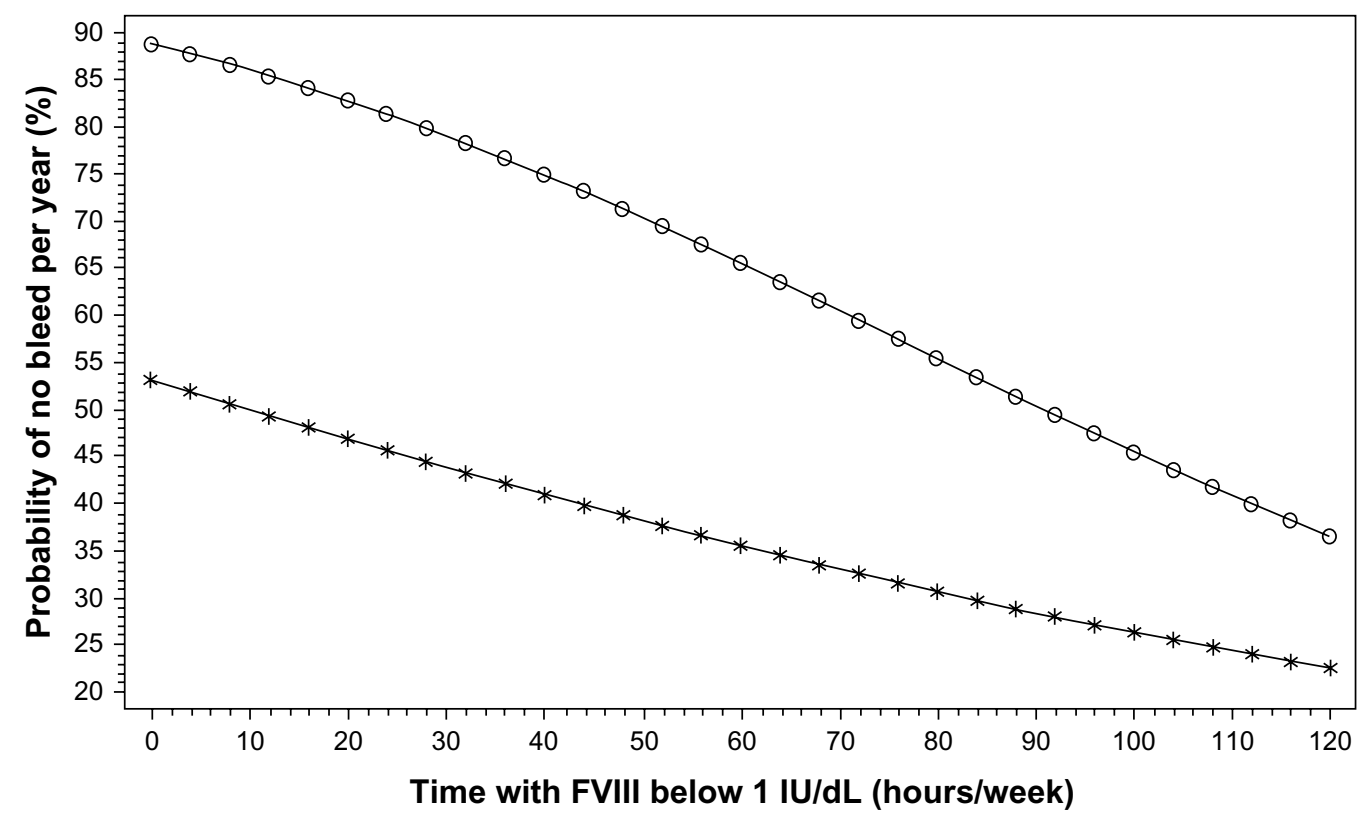

Figure 2 Predicted annual joint bleed rate as a function of time spent with factor $(\mathrm{F}) \mathrm{VIII}<\mathrm{I} I \mathrm{IU} / \mathrm{dL}$. Negative binomial linear model with joint bleed rate as the dependent variable, and time with $\mathrm{FVIII}<\mathrm{I} \mathrm{IU} / \mathrm{dL}$, age, and body weight as independent variables. The figure shows the predicted probability of having no bleeds per year dependent on time per week spent with an FVIII $<$ I IU/dL.

Notes: Open circles $(O)$ represent patients aged I-6 years; asterisks $(*)$ represent patients aged I0-65 years. Reprinted with permission from John Wiley and Sons. Collins PW, Blanchette VS, Fischer K, et al; rAHF-PFM Study Group. Break-through bleeding in relation to predicted factor VIII levels in patients receiving prophylactic treatment for severe hemophilia A. J Thromb Haemost. 2009;7(3):413-420. ${ }^{33}$ Copyright (C) International Society on Thrombosis and Haemostasis. 
spontaneous bleeds, and traumatic bleeds. We conclude from these data that PK-guided prophylaxis is a viable alternative to standard every-other-day prophylaxis: both regimens entailed similar FVIII consumption, whereas the PK-guided regimen required one fewer infusion per week. Both prophylactic regimens resulted in significant reductions in bleed rates: the median ABR was 44 bleeds/ patient/year with episodic treatment vs $0-1$ bleeds/patient/ year with either of the rAHF-PFM prophylaxis regimens. Additionally, rAHF-PFM prophylaxis was associated with a clinically significant improvement in bodily pain and physical functioning, clearly demonstrating HRQOL benefits for this mode of therapy with rAHF-PFM.

\section{Using population PK models to plan routine $\mathrm{PK}$ assessments in the hemophilia clinic}

The data presented thus far indicate that knowledge of individual patient $\mathrm{PKs}$ is critical for determination of the optimal prophylactic dosing for prevention of bleeding. Thus, using a population PK model fitted simultaneously to all available concentration vs time data from our registration studies, we examined relationships between FVIII PKs and factors such as age and body weight, which have been used in the past to approximate PK values. ${ }^{36}$

In the population model, an integrated covariate model describes relationships between the PK parameters and patient characteristics, and an integrated statistical model describes the variance in PK parameters resulting from differences between and within individuals. The model also describes variance due to biologic variability, measurement errors, and errors in the fit of the model to the data. Population PK modeling is especially well suited to analysis of data pooled from separate studies, such as our pivotal, continuation, and pediatric studies ${ }^{14,16,21}$ that used different FVIII infusion and blood sampling schemes; it can also minimize the impact of differences in blood sampling schemes on the PK results. Other researchers also have noted that data from a population PK model may be applied to the real-world setting by indicating the key blood sampling times that are needed for accurate PK estimates, thus reducing sampling to a level that is practical in the setting of routine clinical practice. ${ }^{37-41}$

Our population PK model ${ }^{36}$ used FVIII PK data generated from 236 infusions of rAHF-PFM in 152 patients

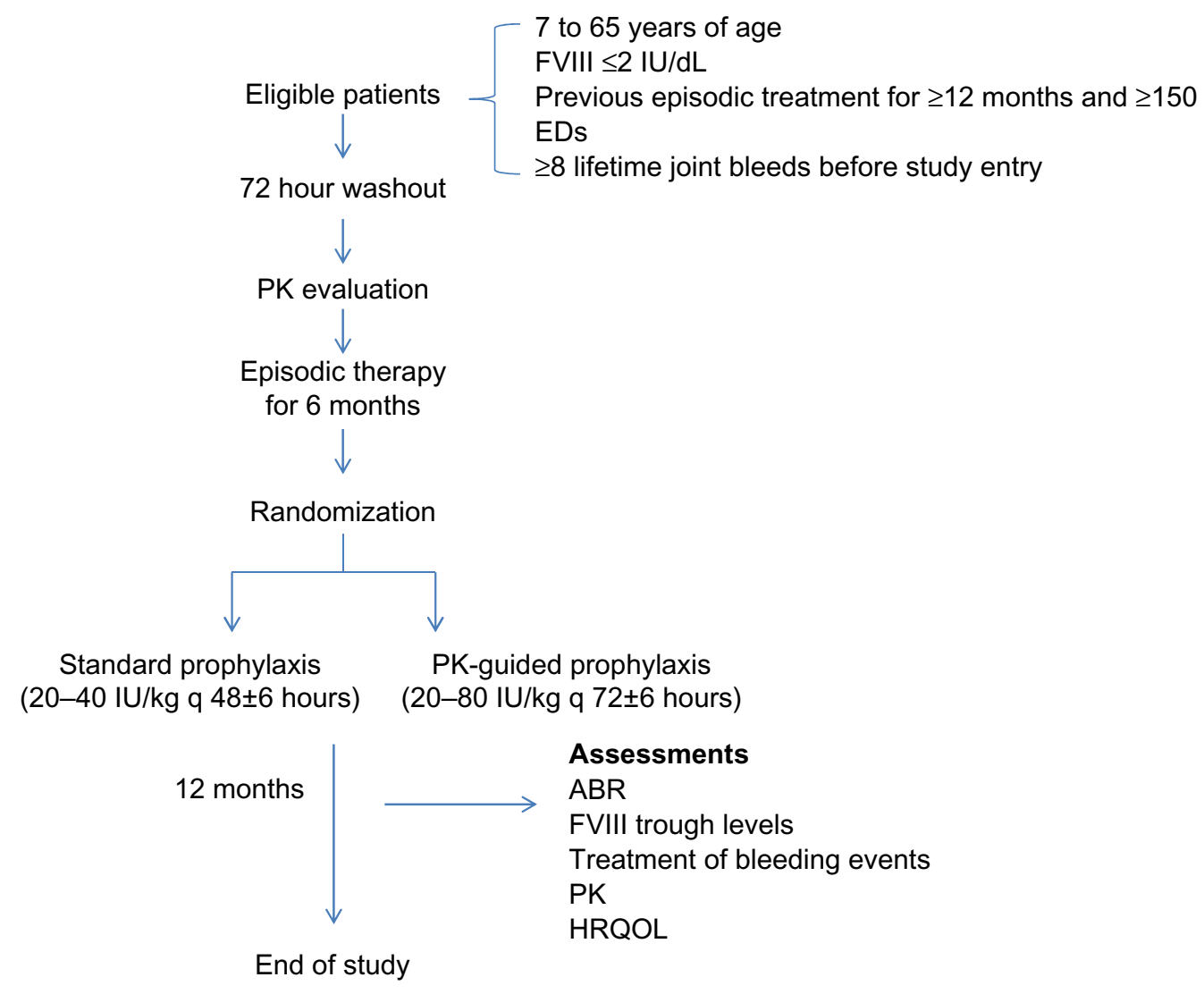

Figure 3 Study design for comparison of standard prophylaxis, PK-guided prophylaxis, and episodic treatment with rAHF-PFM.

Abbreviations: ABR, annual bleed rate (bleeds/patient/year); EDs, exposure days; HRQOL, health-related quality of life; PK, pharmacokinetic; FVIII, factor VIII; rAHF-PFM, recombinant antihemophilic factor produced using a plasma/albumin-free method; q, every. 
(1-65 years of age) with moderately severe to severe hemophilia A from three clinical trials of rAHF-PFM. The final two-compartment model with an additive residual error model, age, as a covariate for $\mathrm{CL}$, and $\mathrm{V}_{\mathrm{ss}}$ similar to the expected plasma volume of the patient, was found to fit the data for both younger and older patients. This finding is in accord with PK modeling data based on other large databases for FVIII treatment. ${ }^{37,39,41,42}$ The FVIII levels predicted by the model were in close agreement with observed levels of FVIII in patients. Notably, the model confirmed the age-associated increase in FVIII $t_{1 / 2}$ and ageassociated decrease in FVIII CL observed with standard PK analyses; the changes were described as continuous functions. Because $t_{1 / 2}$ changes with age, the prophylactic dose required to maintain the FVIII trough level $>1 \mathrm{IU} / \mathrm{dL}$ (or other threshold) will also change and will need to be based on the measured $t_{1 / 2}$ at any given time resulting from the variation in this parameter between patients. The IVR predicted by the model was similar for younger and older patients, in contrast to results obtained using the standard linear regression model where IVR was lower for young children than that for adolescents and adults. In the population model, reduction of blood sampling from eleven to five samples did not alter the estimated PK parameters. The ability of the population PK model to reasonably predict PK parameters of an individual using fewer samples was further tested by comparing the parameters derived using the full PK schedule to the results derived from the population PK model using only one, two, or three samples from the same patient datasets. ${ }^{43}$ As expected, variance between the estimated parameters was small. To externally test the model, an independent analysis was performed using individual subject data from the rAHF-PFM prophylaxis study, ${ }^{17}$ which were not included in the original population PK model. Twocompartment curve fitting of data obtained from a full PK schedule on the rAHF-PFM prophylaxis study showed good agreement with individual PK estimates generated using the population PK model based on data from only four PK samples for subjects on the rAHF-PFM prophylaxis study. ${ }^{44}$ These results demonstrate that a population PK model may be a useful platform for determining an individual's FVIII PK characteristics needed to design an appropriate PK-guided dose and infusion schedule in routine clinical practice in all age groups. The need for fewer blood samples should increase patient convenience, limit discomfort especially in young children - and reduce costs related to handling and assaying of blood samples and extended or multiple clinic visits. The remaining missing link to use in routine practice is a simple way to calculate the individuals PK parameters using the minimal blood samples.

\section{Future directions}

Our data support the advent of population PK-guided prophylaxis as the new treatment paradigm. Whereas we have shown that the use of only a few postinfusion PK samples is adequate for determination of a patient's PK profile, adoption of this method is currently limited by the practitioner's lack of access to population PK data and simple methods for calculating dosing based on such data. The development of an easy to use electronically-based, "PK-calculator" would fill an important need.

The process of identifying the optimal treatment regimen for an individual patient is likely to be complex for some patients. We recently examined the relationship between variables such as average maximal plasma concentration $\left(\mathrm{C}_{\text {max }}\right)$, area under the curve (AUC)/week, time spent above 5, 10, 20, 30, and 40 IU/dL FVIII (ie, peak FVIII levels in the nonhemophilic range), and risk for bleeding in 34 patients receiving PK-guided prophylactic therapy every third day. ${ }^{45}$ This analysis showed a significant relationship between higher $\mathrm{C}_{\max }$ values and/or time spent within the peak, nonhemophilic FVIII range (eg, >30 IU/dL), and lower ABR on every third day dosing regimens. We also noted that higher ABRs during PK-guided prophylaxis were correlated with higher ABRs during the preceding episodic treatment period and with lower percentage reductions in ABR during prophylactic relative to episodic treatment. These data suggest that targeting FVIII trough at $>1 \mathrm{IU} / \mathrm{dL}$ may not be suitable for all patients, especially those with a recent history of high ABR during episodic therapy. Such patients may require alternative dosing regimens including higher doses and/or shorter dose intervals to achieve higher troughs and/or more frequent peaks. Further investigation in this area is warranted, particularly regarding the potential relationship between patient activity levels and the risk of bleeding at various FVIII peak and trough levels.

We also have recently begun to assess the relationships between ABR and HRQOL. Previously, we found that physical HRQOL scores of patients with hemophilia A were lower than those of individuals either in the general population or with chronic back pain. ${ }^{46}$ We and other researchers have reported a positive correlation between prophylactic therapy and improved HRQOL in both children and adults. ${ }^{17,20,46-49}$ However, no analysis has systematically quantified the level of bleeding that results in a negative impact on HRQOL. As a first step in understanding such relationships, we have conducted 
a large multinational, cross-sectional survey to assess health outcomes of children with severe hemophilia A (mean age, 9.7 years) according to reported ABR. ${ }^{50}$ Approximately half of the surveyed children were being treated by episodic therapy and half were treated on a prophylactic regimen. In this survey population, $\mathrm{ABR} \geq 3.0$ was significantly correlated with worsening of both physical and emotional HRQOL scores $(P<0.05)$ and with increasing numbers of target joints and missed school days. These data suggest that even three to four bleeds per year may have a negative impact on a pediatric patient's joint health, school attendance and HRQOL. This result is consistent with results of a study by Funk et $\mathrm{al},{ }^{51}$ in which patients with three or more bleeds per year had increased radiologic joint scores relative to patients with zero to two bleeds per year. Thus, efforts to tailor therapy toward a zero ABR among pediatric patients with severe hemophilia A may improve a patient's quality of life as well as clinical outcomes.

Finally, currently available data has suggested that some patients experience detrimental subclinical bleeding: such patients have low joint ABRs, but show evidence of joint damage on radiologic assessments. ${ }^{10,52}$ These observations suggest the need for long-term joint outcome studies based on radiologic assessment, even in patients with low ABRs. Such studies could also examine the feasibility of measuring indicators of bone and cartilage formation/degradation as surrogate measures of clinical disease progression.

\section{Conclusion}

The development of FVIII concentrates that have virtually no risk of transmission of animal or human blood-borne pathogens has allowed the hemophilia treatment community to shift its focus from concentrate safety to optimization of therapy for prevention of long-term morbidity. Prophylactic dosing, in which FVIII concentrate is infused at regular intervals before the onset of bleeding, is now considered the standard of care for children with severe hemophilia, as well as for adults who experience frequent bleeds. Our analyses of PK, trough levels, prophylactic dosing regimens, and bleeding frequencies show that patient age, FVIII $t_{1 / 2}$, dosing frequency, and adherence to the dosing frequency are important factors that affect the time a patient spends with plasma FVIII $<1$ IU/dL. The commonly used M-W-F dosing regimen has the potential to result in FVIII levels below the bleeding threshold in many patients, if dosing is based only on body weight. PK-guided dosing, however, can be applied to tailor the appropriate dose within a safe and effective range for many patients. Data from rAHF-PFM studies demonstrated that PK-guided prophylactic regimens based on a Bayesian population PK modeling analysis of individual patient data using limited sampling points could be effective in maintaining FVIII trough levels above an individual's threshold for bleeding and may reduce the number of required weekly infusions in some patients. This may in turn, improve adherence and patient HRQOL. Because PK-guided individualized therapy, on the whole, has the potential to be more cost-effective for many patients, while still achieving an ABR of 0-1 bleeds/patient/year, it can also be used to optimize the dosing regimens of those who are not able to achieve this ultimate goal using their conventional, suboptimal body weight-based dosing regimen. More judicious use of FVIII may also expand access to prophylactic therapy, particularly in countries with cost constraints or limited health resources. Finally, our aspiration toward a bleed-free world will require continual vigilance; the choice of regimens should be dynamic, as optimal dose and dosing frequency will likely change as patients age and change lifestyle/physical activity and exhibit changes in FVIII PK. ${ }^{29,53-55}$ With continued research, we will also learn other ways to further optimize therapy and prevent the damage from bleeds before they occur.

\section{Acknowledgment}

The authors thank Martha Sensel PhD, an independent consultant, for providing excellent medical writing support, which was funded by Baxter Healthcare Corporation.

\section{Disclosure}

Bruce M Ewenstein, Gerald Spotts and Lisa Patrone are employees of Baxter Healthcare Corporation. Erik Berntorp has received research grants and honoraria for Advisory Boards and lectures from Baxter Healthcare Corporation.

\section{References}

1. Baker JR, Riske B, Drake JH, et al. US Hemophilia Treatment Center population trends 1990-2010: patient diagnoses, demographics, health services utilization. Haemophilia. 2013;19(1):21-26.

2. Soucie JM, Evatt B, Jackson D. Occurrence of hemophilia in the United States. The Hemophilia Surveillance System Project Investigators. Am J Hematol. 1998;59(4):288-294.

3. Nilsson IM, Hedner U, Ahlberg A. Haemophilia prophylaxis in Sweden Acta Paediatr Scand. 1976;65(2):129-135.

4. Nilsson IM, Blomback M, Ahlberg A. Our experience in Sweden with prophylaxis on haemophilia. Bibl Haematol. 1970;34:111-124.

5. Pettersson H, Nilsson IM, Hedner U, Norehn K, Ahlberg A. Radiologic evaluation of prophylaxis in severe haemophilia. Acta Paediatr Scand. 1981;70(4):565-570

6. Nilsson IM, Berntorp E, Lofqvist T, Pettersson H. Twenty-five years' experience of prophylactic treatment in severe haemophilia A and B. $J$ Intern Med. 1992;232(1):25-32.

7. Fischer K, van der Bom JG, Mauser-Bunschoten EP, et al. The effects of postponing prophylactic treatment on long-term outcome in patients with severe hemophilia. Blood. 2002;99(7):2337-2341. 
8. Fischer K, van der Bom JG, Molho P, et al. Prophylactic versus ondemand treatment strategies for severe haemophilia: a comparison of costs and long-term outcome. Haemophilia. 2002;8(6):745-752.

9. Hay CR. Prophylaxis in adults with haemophilia. Haemophilia. 2007; 13 Suppl s2:10-15.

10. Manco-Johnson MJ, Abshire TC, Shapiro AD, et al. Prophylaxis versus episodic treatment to prevent joint disease in boys with severe hemophilia. N Engl J Med. 2007;357(6):535-544.

11. Collins P, Faradji A, Morfini M, Enriquez MM, Schwartz L. Efficacy and safety of secondary prophylactic vs. on-demand sucrose-formulated recombinant factor VIII treatment in adults with severe hemophilia A: results from a 13-month crossover study. J Thromb Haemost. 2010;8(1):83-89.

12. Ahnstrom J, Berntorp E, Lindvall K, Björkman S. A 6-year follow-up of dosing, coagulation factor levels and bleedings in relation to joint status in the prophylactic treatment of haemophilia. Haemophilia. 2004;10(6):689-697.

13. Björkman S. Prophylactic dosing of factor VIII and factor IX from a clinical pharmacokinetic perspective. Haemophilia. 2003;9 Suppl s1: 101-110.

14. Blanchette VS, Shapiro AD, Liesner RJ, et al. Plasma and albuminfree recombinant factor VIII: pharmacokinetics, efficacy and safety in previously treated pediatric patients. J Thromb Haemost. 2008;6(8): $1319-1326$.

15. Fischer K, Collins P, Björkman S, et al. Trends in bleeding patterns during prophylaxis for severe haemophilia: observations from a series of prospective clinical trials. Haemophilia. 2011;17(3):433-438.

16. Tarantino MD, Collins PW, Hay CR, et al. Clinical evaluation of an advanced category antihaemophilic factor prepared using a plasma/albumin-free method: pharmacokinetics, efficacy, and safety in previously treated patients with haemophilia A. Haemophilia. 2004;10(5): 428-437.

17. Valentino LA, Mamonov V, Hellmann A, et al. A randomized comparison of two prophylaxis regimens and a paired comparison of on-demand and prophylaxis treatments in hemophilia A management. $J$ Thromb Haemost. 2012;10(3):359-367.

18. Auerswald G, Thompson AA, Recht M, et al. Experience of Advate rAHF-PFM in previously untreated patients and minimally treated patients with haemophilia A. Thromb Haemost. 2012;107(6): 1072-1082.

19. Oldenburg J, Goudemand J, Valentino L, et al. Postauthorization safety surveillance of ADVATE [antihaemophilic factor (recombinant), plasma/albumin-free method] demonstrates efficacy, safety and lowrisk for immunogenicity in routine clinical practice. Haemophilia. 2010;16(6):866-877.

20. Pollmann H, Klamroth R, Vidovic N, et al. Prophylaxis and quality of life in patients with hemophilia A during routine treatment with ADVATE [antihemophilic factor (recombinant), plasma/albumin-free method] in Germany: a subgroup analysis of the ADVATE PASS post-approval, non-interventional study. Ann Hematol. 2013;92(5):689-698.

21. Shapiro AD. Anti-hemophilic factor (recombinant), plasma/ albumin-free method (octocog-alpha; ADVATE) in the management of hemophilia A. Vasc Health Risk Manag. 2007;3(5): $555-565$.

22. Fischer K, van der Bom JG, Mauser-Bunschoten EP, et al. Changes in treatment strategies for severe haemophilia over the last 3 decades: effects on clotting factor consumption and arthropathy. Haemophilia. 2001;7(5):446-452.

23. Fijnvandraat $\mathrm{K}$, Peters $\mathrm{M}$, ten Cate JW. Inter-individual variation in half-life of infused recombinant factor VIII is related to pre-infusion von Willebrand factor antigen levels. Br J Haematol. 1995;91(2): 474-476.

24. Kessler CM, Gill JC, White GC 2nd, et al. B-domain deleted recombinant factor VIII preparations are bioequivalent to a monoclonal antibody purified plasma-derived factor VIII concentrate: a randomized, threeway crossover study. Haemophilia. 2005;11(2):84-91.

25. Morfini M. Pharmacokinetics of factor VIII and factor IX. Haemophilia. 2003;9 Suppl s1:94-100.
26. van Dijk K, van der Bom JG, Lenting PJ, et al. Factor VIII half-life and clinical phenotype of severe hemophilia A. Haematologica. 2005;90(4): 494-498.

27. Björkman S, Blanchette VS, Fischer K, et al; Advate Clinical Program Group. Comparative pharmacokinetics of plasma- and albumin-free recombinant factor VIII in children and adults: the influence of blood sampling schedule on observed age-related differences and implications for dose tailoring. J Thromb Haemost. 2010;8(4):730-736.

28. Carlsson M, Berntorp E, Bjorkman S, Lindvall K. Pharmacokinetic dosing in prophylactic treatment of hemophilia A. Eur J Haematol. 1993;51(4):247-252.

29. Franchini M, Mannucci PM. Prophylaxis for adults with haemophilia: towards a personalised approach? Blood Transfus. 2012;10(2): $123-124$.

30. Shapiro AD, Korth-Bradley J, Poon MC. Use of pharmacokinetics in the coagulation factor treatment of patients with haemophilia. Haemophilia. 2005;11(6):571-582.

31. Kasper CK, Dietrich SL, Rapaport SI. Hemophilia prophylaxis with factor VIII concentrate. Arch Intern Med. 1970;125(6):1004-1009.

32. Schimpf K, Fischer B, Rothmann P. Hemophilia A prophylaxis with factor VIII concentrate in a home-treatment program: a controlled study. Scand J Haematol Suppl. 1977;30:79-80.

33. Collins PW, Blanchette VS, Fischer K, et al; rAHF-PFM Study Group. Break-through bleeding in relation to predicted factor VIII levels in patients receiving prophylactic treatment for severe hemophilia A. J Thromb Haemost. 2009;7(3):413-420.

34. Collins PW, Björkman S, Fischer K, et al. Factor VIII requirement to maintain a target plasma level in the prophylactic treatment of severe hemophilia A: influences of variance in pharmacokinetics and treatment regimens. J Thromb Haemost. 2010;8(2):269-275.

35. Lindvall K, Astermark J, Bjorkman S, et al. Daily dosing prophylaxis for haemophilia: a randomized crossover pilot study evaluating feasibility and efficacy. Haemophilia. 2012;18(6):855-859.

36. Björkman S, Oh M, Spotts G, et al. Population pharmacokinetics of recombinant factor VIII: the relationships of pharmacokinetics to age and body weight. Blood. 2012;119(2):612-618.

37. Björkman S. Limited blood sampling for pharmacokinetic dose tailoring of FVIII in the prophylactic treatment of haemophilia A. Haemophilia. 2010;16(4):597-605.

38. Björkman S. Evaluation of the TCIWorks Bayesian computer program for estimation of individual pharmacokinetics of FVIII. Haemophilia. 2011;17(1):e239-e240.

39. Bolon-Larger M, Chamouard V, Bressolle F, Boulieu R. A limited sampling strategy for estimating individual pharmacokinetic parameters of coagulation factor VIII in patients with hemophilia A. Ther Drug Monit. 2007;29(1):20-26.

40. Collins PW, Fischer K, Morfini M, Blanchette VS, Björkman S. Implications of coagulation factor VIII and IX pharmacokinetics in the prophylactic treatment of haemophilia. Haemophilia. 2011;17(1): $2-10$.

41. Stass H. Determination of minimal sampling time points for reliable pharmacokinetic evaluation of recombinant factor VIII: an exploratory population pharmacokinetic analysis in paediatric patients suffering from severe haemophilia. Haemophilia. 2006;12(Suppl 4): $50-55$.

42. Bjorkman S, Folkesson A, Jonsson S. Pharmacokinetics and dose requirements of factor VIII over the age range 3-74 years: a population analysis based on 50 patients with long-term prophylactic treatment for haemophilia A. Eur J Clin Pharmacol. 2009;65(10):989-998.

43. Oh M, Björkman S, Schroth P, et al. Use of a population pharmacokinetic model of ADVATE In pediatric and adult patients with hemophilia A permits limited blood sampling for individual dose tailoring. Blood. 2010;116(21):608.

44. Oh M, Björkman S, Schroth P, et al. Evaluation of a population pharmacokinetic model of a recombinant FVIII (ADVATE) in patients with hemophilia A using external data. JThromb Haemost. 2011;9(Suppl s2) Abstract P-WE-496:676. 
45. Valentino L, Collins P, Pipe S, et al. Peak FVIII levels and time spent in hemostatically effective FVIII range post-infusion correlates with improved efficacy for prophylaxis in hemophilia $\mathrm{A}$ : A closer look at the other end of the curve. Presented at the XXIV Congress of The International Society on Thrombosis and Haemostasis June 29-July 4, 2013, Amsterdam, Netherlands. Available from: http://www.eventure-online.com/eventure/publicAbstractView. do? $\mathrm{id}=217806 \&$ congressId=6839. Accessed October 11, 2013.

46. Klamroth R, Pollmann H, Hermans C, et al. The relative burden of haemophilia A and the impact of target joint development on healthrelated quality of life: results from the ADVATE Post-Authorization Safety Surveillance (PASS) study. Haemophilia. 2011;17(3): $412-421$

47. Duncan N, Shapiro A, Ye X, Epstein J, Luo MP. Treatment patterns, health-related quality of life and adherence to prophylaxis among haemophilia A patients in the United States. Haemophilia. 2012;18(5): 760-765.

48. Gringeri A, von Mackensen S, Auerswald G, et al. Health status and health-related quality of life of children with haemophilia from six West European countries. Haemophilia. Mar 2004;10 Suppl 1:26-33.

49. Naraine VS, Risebrough NA, Oh P, et al. Health-related quality-of-life treatments for severe haemophilia: utility measurements using the Standard Gamble technique. Haemophilia. 2002;8(2):112-120.
50. Valentino L, Giangrande P, Epstein J, Xiong Y, Ito D, Li-McLeod J. The relationship between specific annual bleed rates and health outcomes among children with severe hemophilia A. Presented at the $X X I V$ Congress of The International Society on Thrombosis and Haemostasis June 29-July 4, 2013. Available from: http://www.eventure-online. com/eventure/publicAbstractView.do? $\mathrm{id}=218067 \&$ congress $I d=6839$. Accessed October 11, 2013.

51. Funk MB, Schmidt H, Becker S, et al. Modified magnetic resonance imaging score compared with orthopaedic and radiological scores for the evaluation of haemophilic arthropathy. Haemophilia. 2002;8(2): 98-103.

52. Lundin B, Babyn P, Doria AS, et al; International Prophylaxis Study Group. Compatible scales for progressive and additive MRI assessments of haemophilic arthropathy. Haemophilia. 2005;11(2):109-115.

53. Richards M, Altisent C, Batorova A, et al. Should prophylaxis be used in adolescent and adult patients with severe haemophilia? An European survey of practice and outcome data. Haemophilia. 2007;13(5):473-479.

54. van Dijk K, Fischer K, van der Bom JG, Scheibel E, Ingerslev J, van den Berg HM. Can long-term prophylaxis for severe haemophilia be stopped in adulthood? Results from Denmark and The Netherlands. $\mathrm{Br}$ J Haematol. 2005;130(1):107-112.

55. Walsh CE, Valentino LA. Factor VIII prophylaxis for adult patients with severe haemophilia A: results of a US survey of attitudes and practices. Haemophilia. 2009;15(5):1014-1021.
Biologics: Targets \& Therapy

\section{Publish your work in this journal}

Biologics: Targets \& Therapy is an international, peer-reviewed journal focusing on the patho-physiological rationale for and clinical application of Biologic agents in the management of autoimmune diseases, cancers or other pathologies where a molecular target can be identified. This journal is indexed on PubMed Central, CAS, EMBase, Scopus

\section{Dovepress}

and the Elsevier Bibliographic databases. The manuscript management system is completely online and includes a very quick and fair peerreview system, which is all easy to use. Visit http://www.dovepress. com/testimonials.php to read real quotes from published authors. 